\title{
THE LIMIT CYCLES \\ OF A CLASS OF QUINTIC POLYNOMIAL VECTOR FIELDS
}

\author{
JAUME LLIBRE $^{1}$ AND TAYEB SALHI ${ }^{2}$
}

\begin{abstract}
Using the inverse integrating factor we study the limit cycles of a class of polynomial vector fields of degree 5 .
\end{abstract}

\section{Introduction AND STATEMENT OF the MAin RESUlts}

One of the main problems in the qualitative theory of differential equations is the study of the limit cycles of planar differential systems and specially of the planar polynomial differential systems, see for instance the book of Ye Yanqian et al. [17] dedicated only to study the limit cycles, mainly of the polynomial differential systems of degree 2 . The main interest for studying the limit cycles of the planar polynomial differential systems is due to the 16-th Hilbert problem, see for instance [13] and [15]. Many recent papers are also dedicated to the study of the limit cycles, see for instance the papers $[2,3,6,12,16]$ which are more related with our present work.

In this paper we shall study the limit cycles of a class of two-dimensional autonomous polynomial differential systems of the form

$$
\begin{aligned}
& \dot{x}=P(x, y), \\
& \dot{y}=Q(x, y),
\end{aligned}
$$

where

$$
\begin{aligned}
& P(x, y)=\lambda x+2 a b y+P_{3}(x, y)+P_{5}(x, y), \\
& Q(x, y)=-2 a b x+\lambda y+Q_{3}(x, y)+Q_{5}(x, y),
\end{aligned}
$$

and $P_{3}, Q_{3}, P_{5}$ and $Q_{5}$ are homogeneous polynomials of degree $3,3,5$ and 5 respectively.

Probably the most difficult and, in general, unsolved problem for planar differential systems is the determination of their limit cycles. We recall that a limit cycle is an isolated periodic solution in the set of all periodic solutions of system (1).

In 1996 Giacomini, Llibre and Viano [6] introduced a new method for studying the existence and nonexistence of limit cycles of planar vector fields. This method is based on the following result.

2010 Mathematics Subject Classification. 34C29, 34C25, 47H11. field.

Key words and phrases. limit cycle, periodic orbit, inverse integrating factor, polynomial vector

* The first author is partially supported by a MCYT/FEDER grant MTM2008-03437, by a CIRIT grant number 2009SGR-410 and by ICREA Academia. The second author is partially supported by the Algerian Ministry of Higher Education and Scientific Research under project B03320090002. 
Theorem 1. Let (1) be a $C^{1}$ differential system defined in the open subset $U$ of $\mathbb{R}^{2}$. Let $V=V(x, y)$ be a $C^{1}$ solution of the linear partial differential equation

$$
P \frac{\partial V}{\partial x}+Q \frac{\partial V}{\partial y}-\left(\frac{\partial P}{\partial x}+\frac{\partial Q}{\partial y}\right) V=0
$$

defined in $U$. If $\gamma$ is a limit cycle of the differential system (1), then $\gamma$ is contained in $\Sigma=\{(x, y) \in U: V(x, y)=0\}$.

For an easy proof of Theorem 1 see [16].

In fact the function $V(x, y)$ is the inverse of an integrating factor of the differential system (1). This function has also been used for studying the limit cycles bifurcating from a center, Hamiltonian or not, see $[7,8,9]$; or the semistable limit cycles see [10]. For a recent summary of the applications of the inverse integrating function $V$ see [5].

Here we shall study the limit cycles of the following 3-parametric class of quintic polynomial differential systems:

$$
\begin{aligned}
\dot{x}= & \lambda x+2 a b y-\frac{\lambda(a+1)}{a} x^{3}-2(-a+b+2 a b) x^{2} y-\frac{\lambda(b+1)}{b} x y^{2}-4 a b y^{3} \\
& +\frac{\lambda}{a} x^{5}-2(a-2 b) x^{4} y+\frac{\lambda(a+b)}{a b} x^{3} y^{2}+4 b x^{2} y^{3}+\frac{\lambda}{b} x y^{4}+2 a y^{5}, \\
\dot{y}= & -2 a b x+\lambda y+4 a b x^{3}-\frac{\lambda(1+a)}{a} x^{2} y+2(a-b+2 a b) x y^{2}-\frac{\lambda(1+b)}{b} y^{3} \\
& -2 b x^{5}+\frac{\lambda}{a} x^{4} y-4 a x^{3} y^{2}+\frac{\lambda(a+b)}{a b} x^{2} y^{3}-2(2 a-b) x y^{4}+\frac{\lambda}{b} y^{5} .
\end{aligned}
$$

The parameters are $\lambda, a$ and $b$ with $a b \neq 0$.

We denote by $O$ the equilibrium point located at the origin of coordinates of system (3).

Let $\gamma$ be a limit cycle of system (3). The stable manifold of $\gamma$ is formed by all the orbits of system (3) having as $\omega$-limit the limit cycle $\gamma$. Similarly, the unstable manifold of $\gamma$ is formed by all the orbits of system (3) having as $\alpha$-limit the limit cycle $\gamma$. For a definition of $\omega$-limit and $\alpha$-limit see for instance the Chapter 1 of [4].

Our main result on the limit cycles of system (3) is the following one.

Theorem 2. For the polynomial differential system (3) with $a b \neq 0$ the following statements hold.

(a) If $\lambda=0$, then system (3) has no limit cycles.

(b) If $a<0$ and $0<b \leq 1$, or $0<a \leq 1$ and $b<0$, or $a \geq 1$ and $0<b \leq 1$, or $0<a \leq 1$ and $b \geq 1$, then system (3) has no limit cycles.

(c) Assume $\lambda \neq 0$. If either $a<0$ and $b>1$, or $a>1$ and $b<0$, then system (3) has exactly one limit cycle $\gamma$ surrounding the origin $O$. Moreover, the stable (respectively unstable) manifold of $\gamma$ is $\mathbb{R}^{2} \backslash\{O\}$ if $\lambda>0$ (respectively $\lambda<0)$.

(d) Assume $\lambda \neq 0$. If $a<0$ and $b<0$, then system (3) has at most one limit cycle.

(e) If $\lambda \neq 0,0<a<1$ and $0<b<1$, then system (3) has exactly two limit cycles $\gamma_{1}$ and $\gamma_{2}$. Assume that $R_{k}$ is the bounded open region limited by $\gamma_{k}$, and that $\gamma_{1} \subset R_{2}$. Then, the stable (respectively unstable) manifold of 
$\gamma_{1}$ is $R_{2} \backslash\{O\}$ if $\lambda>0$ (respectively $\lambda<0$ ), and the unstable (respectively stable) manifold of $\gamma_{2}$ is $\mathbb{R}^{2} \backslash\left\{\mathrm{Cl}\left(R_{1}\right)\right\}$ if $\lambda>0$ (respectively $\lambda<0$ ).

(f) If $\lambda \neq 0, a>1$ and $b>1$, then system (3) has at most two limit cycles.

Theorem 2 is proved in section 3 . We note that the statements of Theorem 2 covers all the the possible values for the parameters $\lambda, a, b \in \mathbb{R}$ with $a b \neq 0$.

More important than the Theorem 2 itself, are the tools for proving it that can be applied to other classes of planar differential systems.

We believe that the following two open questions have a positive answer.

Open Question 1. Is it true that the polynomial differential system (3) under the assumptions of statement (d) of Theorem 2 has exactly one limit cycle?

Open Question 2. Is it true that the polynomial differential system (3) under the assumptions of statement ( $f$ ) of Theorem 2 has exactly two limit cycles?

In the next section we recall the basic results of the Poincaré compactification of a polynomial differential system in the plane, for more details see for instance [11], or the Chapter 5 of [4]. In particular we describe the dynamics of the polynomial differential system (3) in a neighborhood of the infinity.

In this paper we have used ideas of Chavarriga, Giacomini and Giné [3].

In the next section we characterize the dynamics of the polynomial differential system (3) in a neighborhood of the infinity.

\section{Poincaré compactification}

Let $X=(P, Q) \in \mathcal{P}_{n}\left(\mathbb{R}^{2}\right)$ be any planar polynomial vector field of degree $n$, i.e. $P$ and $Q$ are polynomials in the variables $x$ and $y$ such that the maximum degree of the degrees of $P$ and $Q$ is $n$. The Poincaré compactified vector field $p(X)$ corresponding to $X$ is an analytic vector field induced on the 2 -dimensional sphere $\mathbb{S}^{2}$ as follows.

Let $\mathbb{S}^{2}=\left\{y=\left(y_{1}, y_{2}, y_{3}\right) \in \mathbb{R}^{3}: y_{1}^{2}+y_{2}^{2}+y_{3}^{2}=1\right\}$ (the Poincaré sphere) and $T_{y} \mathbb{S}^{2}$ be the tangent space to $\mathbb{S}^{2}$ at point $y$. Consider the central projection $f$ : $T_{(0,0,1)} \mathbb{S}^{2} \rightarrow \mathbb{S}^{2}$. This map defines two copies of $X$, one in the northern hemisphere and the other in the southern hemisphere. Denote by $X^{\prime}$ the vector field $D f \circ X$ defined on $\mathbb{S}^{2}$ except on its equator $\mathbb{S}^{1}=\left\{y \in \mathbb{S}^{2}: y_{3}=0\right\}$. Clearly $\mathbb{S}^{1}$ is identified to the infinity of $\mathbb{R}^{2}$. In order to extend $X^{\prime}$ to a vector field on $\mathbb{S}^{2}$ (including $\mathbb{S}^{1}$ ) it is necessary that $X$ satisfies suitable conditions. In the case that $X \in \mathcal{P}_{n}\left(\mathbb{R}^{2}\right), p(X)$ is the only analytic extension of $y_{3}^{n-1} X^{\prime}$ to $\mathbb{S}^{2}$. On $\mathbb{S}^{2} \backslash \mathbb{S}^{1}$ there are two symmetric copies of $X$, and knowing the behavior of $p(X)$ around $\mathbb{S}^{1}$, we know the behavior of $X$ at infinity. The projection of the closed northern hemisphere of $\mathbb{S}^{2}$ on $y_{3}=0$ under $\left(y_{1}, y_{2}, y_{3}\right) \longmapsto\left(y_{1}, y_{2}\right)$ is called the Poincaré disc, and it is denoted by $\mathbb{D}^{2}$. The Poincaré compactification has the property that $\mathbb{S}^{1}$ is invariant under the flow of $p(X)$.

In this paper we say that two polynomial vector fields $X$ and $Y$ on $\mathbb{R}^{2}$ are topologically equivalent if there exists a homeomorphism on $\mathbb{S}^{2}$ preserving the infinity $\mathbb{S}^{1}$ carrying orbits of the flow induced by $p(X)$ into orbits of the flow induced by $p(Y)$, preserving or reversing simultaneously the sense of all orbits.

As $\mathbb{S}^{2}$ is a differentiable manifold, for computing the expression of $p(X)$, we can consider the six local charts $U_{i}=\left\{y \in \mathbb{S}^{2}: y_{i}>0\right\}$, and $V_{i}=\left\{y \in \mathbb{S}^{2}: y_{i}<0\right\}$ where $i=1,2,3$; and the diffeomorphisms $F_{i}: U_{i} \rightarrow \mathbb{R}^{2}$ and $G_{i}: V_{i} \rightarrow \mathbb{R}^{2}$ for 
$i=1,2,3$ are the inverses of the central projections from the planes tangent at the points $(1,0,0),(-1,0,0),(0,1,0),(0,-1,0),(0,0,1)$ and $(0,0,-1)$ respectively. If we denote by $(u, v)$ the value of $F_{i}(y)$ or $G_{i}(y)$ for any $i=1,2,3$ (so $(u, v$ ) represents different things according to the local charts under consideration), then some easy computations give for the vector field $p(X)$ the following expressions:

$$
\begin{array}{rc}
v^{n} \Delta(u, v)\left(Q\left(\frac{1}{v}, \frac{u}{v}\right)-u P\left(\frac{1}{v}, \frac{u}{v}\right),-v P\left(\frac{1}{v}, \frac{u}{v}\right)\right) & \text { in } \quad U_{1}, \\
v^{n} \Delta(u, v)\left(P\left(\frac{u}{v}, \frac{1}{v}\right)-u Q\left(\frac{u}{v}, \frac{1}{v}\right),-v Q\left(\frac{u}{v}, \frac{1}{v}\right)\right) & \text { in } \quad U_{2}, \\
\Delta(u, v)(P(u, v), Q(u, v)) & \text { in } \quad U_{3},
\end{array}
$$

where $\Delta(u, v)=\left(u^{2}+v^{2}+1\right)^{-\frac{1}{2}(n-1)}$. The expression for $V_{i}$ is the same as that for $U_{i}$ except for a multiplicative factor $(-1)^{n-1}$. In these coordinates for $i=1,2$, $v=0$ always denotes the points of $\mathbb{S}^{1}$. In what follows we omit the factor $\Delta(u, v)$ by rescaling the vector field $p(X)$. Thus we obtain a polynomial vector field in each local chart.

The singular points of the vector field $p(X)$ on the infinity, i.e. on $\mathbb{S}^{1}$ are called the infinite singular points of the vector $X$. Due to the fact that the expression for $V_{i}$ is the same as that for $U_{i}$ except for a multiplicative factor $(-1)^{n-1}$, it follows that the infinite singular points appears by pairs diametrally opposite in the Poincaré disc. Moreover, once we have studied the infinite singular points in the local chart $U_{1}$ and consequently also in the local chart $V_{1}$, only it is necessary to study if the origin of the local chart $U_{2}$ is an infinite singular point.

Proposition 3. The following statements hold for the polynomial differential system (3) with $a b \neq 0$.

(a) If $a b>0$ then the infinity $\mathbb{S}^{1}$ of system (3) is formed by a periodic orbit.

(b) If $a b<0$ then at infinity there are only two pairs of infinite singular points, that in the local chart $U_{1}$ have coordinates $( \pm \sqrt{-b / a}, 0)$. Moreover, these two singular points are saddles.

Proof. Since the infinity $\mathbb{S}^{1}$ is invariant by the flow of the Poincaré compactification $p(X)$, i.e. it is formed by orbits of $p(X)$, in order to proof the statement (a) of the proposition it is sufficient to show that the Poincaré compactification of system (3) has no infinite singular points.

Using (4) we obtain that the Poincaré compactification of system (3) in the local chart $U_{1}$ is given by

$$
\begin{aligned}
\dot{u}= & -2 a u^{6}+4 a b v^{2} u^{4}-2(2 a+b) u^{4}-2 a b v^{4} u^{2}+8 a b v^{2} u^{2}-2(a+2 b) u^{2} \\
& -2 a b v^{4}+4 a b v^{2}-2 b, \\
\dot{v}= & -2 a v u^{5}-\frac{v \lambda u^{4}}{b}+4 a b v^{3} u^{3}-4 b v u^{3}+\frac{(b+1) v^{3} \lambda u^{2}}{b}-\frac{(a+b) v \lambda u^{2}}{a b} \\
& -2 a b v^{5} u+2(2 b a-a+b) v^{3} u+2(a-2 b) v u-v^{5} \lambda+\frac{(a+1) v^{3} \lambda}{a}-\frac{v \lambda}{a} .
\end{aligned}
$$

Then the infinite singular points $\left(u^{*}, 0\right)$ must satisfy that $u^{*}$ is a zero of

$$
\left.\dot{u}\right|_{v=0}=-2\left(1+u^{2}\right)^{2}\left(b+a u^{2}\right) .
$$

So $u^{*}= \pm \sqrt{-b / a}$. Consequently they exist if $a b<0$. 
The eigenvalues at the singular point $(\sqrt{-b / a}, 0)$ are

$$
\frac{4}{b}(a-b)^{2}\left(-\frac{b}{a}\right)^{3 / 2}, \quad \frac{2}{a}(a-b)^{2} \sqrt{-\frac{b}{a}},
$$

and at the singular point $(-\sqrt{-b / a}, 0)$ are

$$
\frac{4}{a}(a-b)^{2} \sqrt{-\frac{b}{a}}, \quad \frac{2}{b}(a-b)^{2}\left(-\frac{b}{a}\right)^{3 / 2} .
$$

Since these singular points exist when $a b<0$, it follows that both singular points are saddles, for more details see for instance Theorem 65 of [1], or Theorem 2.19 of $[4]$.

The proposition will be proved if we check that the origin of the local chart $U_{2}$ is not an infinite singular point. Using (5) we obtain that the Poincaré compactification of system (3) in the local chart $U_{2}$ is given by

$$
\begin{aligned}
\dot{u}= & 2 b u^{6}-4 a b v^{2} u^{4}+2(a+2 b) u^{4}+2 a b v^{4} u^{2}-8 a b v^{2} u^{2}+2(2 a+b) u^{2} \\
& +2 a b v^{4}-4 a b v^{2}+2 a, \\
\dot{v}= & 2 b v u^{5}-\frac{v \lambda u^{4}}{a}-4 a b v^{3} u^{3}+4 a v u^{3}+\frac{(a+1) v^{3} \lambda u^{2}}{a}-\frac{(a+b) v \lambda u^{2}}{a b} \\
& +2 a b v^{5} u-2(2 b a+a-b) v^{3} u+2(2 a-b) v u-v^{5} \lambda+\frac{(b+1) v^{3} \lambda}{b}-\frac{v \lambda}{b} .
\end{aligned}
$$

The origin $(0,0)$ of $U_{2}$ is not a singular point because $\left.\dot{u}\right|_{(u=0, v=0)}=2 a \neq 0$. This completes the proof of the proposition.

\section{Proof of Theorem 2}

Let $U$ be an open subset of $\mathbb{R}^{2}$. We recall that a $C^{1}$ function $H: U \rightarrow \mathbb{R}$ is a first integral of the differential system (1) if $H$ is constant on every solution $(x(t), y(t))$ of system (1) contained in $U$; i.e. if $X H=0$ in $U$ where

$$
X=P(x, y) \frac{\partial}{\partial x}+Q(x, y) \frac{\partial}{\partial y} \text {. }
$$

The following result is well known, for a proof see for instance Theorem 3 of Chapter 11 of [14].

Lemma 4. Let $H: U \rightarrow \mathbb{R}$ be a first integral of the differential system (1). Then system (1) has no limit cycles contained in $U$.

Proof of statement (a) of Theorem 2. We define the rational function

$$
H=H(x, y)=\frac{\left(x^{2}+y^{2}\right)\left(x^{2}+y^{2}-1\right)}{\frac{x^{2}}{a}+\frac{y^{2}}{b}-1}
$$

Then it is easy to check that $H$ is a first integral of system (3) with $\lambda=0$ and $a b \neq 0$ defined in

$$
\mathbb{R}^{2} \backslash\left\{(x, y) \in \mathbb{R}^{2}: \frac{x^{2}}{a}+\frac{y^{2}}{b}=1\right\},
$$

and that $1 / H$ is a first integral of system (3) with $\lambda=0$ and $a b \neq 0$ defined in

$$
\mathbb{R}^{2} \backslash\left\{(x, y) \in \mathbb{R}^{2}:\left(x^{2}+y^{2}\right)\left(x^{2}+y^{2}-1\right)=0\right\} .
$$


Since the domains of the definitions of the first integrals $H$ and $1 / H$ cover all the plane $\mathbb{R}^{2}$, by Lemma 4 it follows that system (3) with $\lambda=0$ and $a b \neq 0$ has no limit cycles.

In order to prove the remaining statements of Theorem 2 we shall need the following results.

Lemma 5. The polynomial

$$
V=V(x, y)=\left(x^{2}+y^{2}\right)\left(x^{2}+y^{2}-1\right)\left(\frac{x^{2}}{a}+\frac{y^{2}}{b}-1\right)
$$

is an inverse integrating factor of the polynomial differential system (3) with $a b \neq 0$.

Proof. It is easy to check that the function $V$ given by (6) satisfies equation (2) for the polynomial differential system (3).

Lemma 6. The unique possible limit cycles of the polynomial differential system (3) with $a b \neq 0$ are the unit circle $x^{2}+y^{2}=1$ and the ellipse $\frac{x^{2}}{a}+\frac{y^{2}}{b}=1$ when $a>0$ and $b>0$.

Proof. From (2) we have that $\left.\dot{V}\right|_{V=0}=0$, so the curve $V=0$ given by (6) is formed by orbits of system (3). Two of these orbits do not change with the parameters of systems, are the equilibrium point at the origin $x^{2}+y^{2}=0$, and the unit circle $x^{2}+y^{2}=1$. We note that the conic $\frac{x^{2}}{a}+\frac{y^{2}}{b}=1$ is an ellipse if and only if $a>0$ and $b>0$. Now, Theorem 1 completes the proof of this lemma.

In what follows for the system (3) the equilibrium point at the origin $x^{2}+y^{2}=0$ will be denoted by $O$, the unit circle $x^{2}+y^{2}=1$ will be denoted by $C$, and the conic $\frac{x^{2}}{a}+\frac{y^{2}}{b}=1$ will be denoted by $E$ independently if it is an ellipse or not.

We shall prove the different statements of Theorem 2 .

Proof of statement (f) of Theorem 2. The proof of this statement follows immediately from Lemma 6 .

Proof of statement (d) of Theorem 2. The proof of this statement follows immediately from Lemma 6 taking into account that now the curve $E$ is not an ellipse.

Proof of statement (b) of Theorem 2. We shall study the intersection of the circle $C$ with the curve $E$. Both curves are invariant by the flow of system (3) because they are formed by orbits of this system. Therefore, the intersection points of both curves are either

(7) $\left( \pm \sqrt{\frac{a-a b}{a-b}}, \pm \sqrt{\frac{a b-b}{a-b}}\right)$ if $a(1-b)(a-b) \geq 0, b(a-1)(a-b) \geq 0, a \neq b$,

or the circle $C$ if $a=b=1$. These intersection points are equilibrium points of system (3). It is easy to check that such points are real if and only if the assumptions of statement (b) are satisfied. Consequently under these assumptions on the circle $C$ there are equilibrium points, and on the ellipse $E$ if $a>0$ and $b>0$ also there are equilibrium points. Hence statement (b) is proved.

Lemma 7. The following statements hold for the polynomial differential system (3) with $a b \neq 0$. The origin is an equilibrium point with eigenvalues $\lambda \pm 2 a b i$. 
(a) If $\lambda \neq 0$, then the origin is a focus, stable if $\lambda<0$, and unstable if $\lambda>0$.

(b) If $\lambda=0$, then the origin is a center.

Proof. It follows immediately of the Hartman-Grobman Theorem, see for instance Theorem 65 of [1], or Theorem 2.19 of [4].

Lemma 8. For the polynomial differential system (3) with $a b \neq 0$ the possible equilibrium points on the unit circle $C$ are given by (7), and the possible equilibrium points on the ellipse $E$ when $a>0$ and $b>0$ are also given by (7).

Proof. From the proof of statement (b) we know that the points (7), when they are real, are equilibrium points leaving on the unit circle $C$ and on the conic $E$.

If we calculate the possible equilibrium points of system (3) on the unit circle we only get the points (7).

If we calculate the possible equilibrium points of system (3) on the ellipse $E$ when $a>0$ and $b>0$ we obtain the points (7) and the points

$$
\left( \pm \sqrt{\frac{a b}{b-a}}, \pm \sqrt{\frac{a b}{a-b}}\right) .
$$

Clearly, since $a>0$ and $b>0$ these last four points are never real. So the lemma is proved.

Lemma 9. The origin $O$ is the unique finite equilibrium point of the polynomial differential system (3) with $a b \neq 0$ if either $a<0$ and $b>1$, or $0<a<1$ and $0<b<1$, or $a>1$ and $b<0$.

Proof. We write system (3) in polar coordinates $(r, \theta)$ where $x=r \cos \theta$ and $y=$ $r \sin \theta$. Then we have

$$
\begin{aligned}
& \dot{r}=\frac{r\left(1-r^{2}\right)\left(((-a-b) \lambda+(a-b) \cos (2 \theta) \lambda+2 a(a-b) b \sin (2 \theta)) r^{2}+2 a b \lambda\right)}{2 a b}, \\
& \dot{\theta}=(-a-b+(a-b) \cos (2 \theta)) r^{4}+4 a b r^{2}-2 a b .
\end{aligned}
$$

If we solve $\dot{\theta}=0$ with respect to the variable $r^{2}$ we obtain

$$
r^{2}=\frac{-4 a b \pm \sqrt{16 a^{2} b^{2}+8 a b(-a-b+(a-b) \cos (2 \theta))}}{2(-a-b+(a-b) \cos (2 \theta))} .
$$

In order that the system cannot have other equilibrium points different from the origin we must have

$$
16 a^{2} b^{2}+8 a b(-a-b+(a-b) \cos (2 \theta))<0,
$$

for all $\theta$. It is easy to verify that this is the case for the three regions of the statement of this lemma.

Proof of statement (c) of Theorem 2. From Lemma 6 it follows that the unique possible limit cycle of system (3) under the assumptions of statement (c) is the unit circle $C$. Moreover, by Lemma 8 there are no equilibrium points on $C$. Therefore, since is $C$ is invariant by the flow of system (3), it follows that $C$ is a periodic orbit.

On the other hand $C$ surrounds the origin $O$, which is a focus (see Lemma 7). We can define the Poincaré return map $\pi$ from the positive $x$-axis into itself. Since the differential system (3) is analytic, the map $\pi$ is also analytic (for more details 
see for instance the section 1.6 of [4]). Since the origin is a focus, this Poincaré map $\pi$ cannot be the identity. By analyticity it has at most a discrete number of fixed points, i.e. of periodic orbits. Since they are isolated in the set of all periodic orbits, they are limit cycles. But the unique limit cycle that the system can have is the unit circle $C$. Hence, $C$ is a limit cycle.

In short, in $\mathbb{R}^{2}$ the unique separatrices of system (3) are the equilibrium point at the origin and the limit cycle $C$, see the definition of separatrix in section 1.8 of [4]. Then, using the result of Lemma 7, and the Poincaré-Bendixson Theorem (see Theorem 1.25 of [4]), the proof of statement (c) is completed.

Proof of statement (e) of Theorem 2. From Lemma 6 it follows that the unique possible limit cycle of system (3) under the assumptions of statement (e) are the unit circle $C$ and the ellipse $E$. Moreover, by Lemma 8 there are no equilibrium points on $C$ and on $E$. Therefore, since $C$ and $E$ are invariant by the flow of system (3), it follows that $C$ and $E$ are periodic orbits.

On the other hand $C$ and $E$ surround the origin $O$, which is a focus (see Lemma 7 ). We can define the Poincaré return map $\pi$ from the positive $x$-axis into itself. Since the differential system (3) is analytic, the map $\pi$ is also analytic. Since the origin is a focus, this Poincaré map $\pi$ cannot be the identity. By analyticity it has at most a discrete number of fixed points, i.e. of periodic orbits. Since they are isolated in the set of all periodic orbits, they are limit cycles. But the unique possible limit cycles that the system can have are the unit circle $C$ and the ellipse $E$. Therefore, $C$ and $E$ are limit cycles.

In short, in $\mathbb{R}^{2}$ the unique separatrices of system (3) are the equilibrium point at the origin and the limit cycles $C$ and $E$. Then, using the result of Lemma 7, and the Poincaré-Bendixson Theorem, the proof of statement (e) is completed.

In short we have proved all the statements of Theorem 2 .

We note that the difficulties for proving the two open questions stated in section 1 come from the fact that under the corresponding assumptions of these open questions the differential system (3) have additional equilibrium points, and the global dynamics of the system becomes more difficult to control.

\section{REFERENCES}

[1] A. A. Andronov, E. A. Leontovich, I. I. Gordon, and A. L. Maier, "Qualitative Theory of Second-Order Dynamical Systems", Wiley, New York, 1973.

[2] J. CAO AND H. JiAng, Planar polynomial vector fields having first integrals and algebraic limit cycles, J. Math. Anal. Appl. 361 (2010), 177-186.

[3] J. Chavarriga, H. Giacomini and J. Giné, On a new type of bifurcation of limit cycles for a planar cubic system, Nonlinear Analysis 36 (1999), 139-149.

[4] F. Dumortier, J. Llibre And J.C. Artés, Qualitative theory of planar differential systems, UniversiText, Springer-Verlag, New York, 2006.

[5] I.A. García And M. Grau, A survey on the inverse integrating factor, Qual. Theory Dyn. Syst. 9 (2010), 115-166.

[6] H. Giacomini, J. Llibre and M. Viano, On the nonexistence, existence, and uniquennes of limit cycles, Nonlinearity 9 (1996), 501-516.

[7] H. Giacomini, J. Llibre And M. Viano, On the shape of limit cycles that bifurcate from Hamiltonian centers, Nonlinear Analysis, Theory, Methods and Applications 41 (2001), 523537. 
[8] H. Giacomini, J. Llibre and M. Viano, The shape of limit cycles that bifurcate from nonHamiltonian centers, Nonlinear Analysis, Theory, Methods and Applications 43 (2001), 837859.

[9] H. Giacomini, J. Llibre And M. Viano, Arbitrary order bifurcations for perturbed Hamiltonian planar systems via the reciprocal of an integrating factor, Nonlinear Analysis, Theory, Methods and Applications 48 (2002), 117-136.

[10] H. Giacomini, J. Llibre and M. Viano, Semistable limit cycles that bifurcate from centers, International J. of Bifurcation and Chaos 13 (2003), 3489-3498.

[11] E. A. GonzÁlez, Generic properties of polynomial vector fields at infinity, Trans. Amer. Math. Soc. 143 (1969), 201-222.

[12] M. Han And V.G. Romanovski, Estimating the number of limit cycles in polynomials systems, J. Math. Anal. Appl. 368 (2010), 491-497.

[13] D. Hilbert, Mathematische Problem (lecture), Second Internat. Congress Math. Paris, 1900, Nachr. Ges. Wiss. Göttingen Math.-Phys. Kl. 1900, pp 253-297.

[14] M.W. Hirsch And S. Smale, Differential Equations, Dynamical Systems, and Linear Algebra, Academic Press, New York, 1974.

[15] Yu. S. Ilyashenko, Centennial history of Hilbert's 16th problem, Bull. Amer. Math. Soc. 39 (2002), 301-354.

[16] J. Llibre And G. RodríGuez, Configurations of limit cycles and planar polynomial vector fields, J. Differential Equations 198 (2004), 374-380.

[17] Ye Yanqian et AL., Theory of Limit Cycles, Translations of Math. Monographs, Vol. 66, Amer. Math. Soc, Providence, 1986.

1 Departament de Matematiques, Universitat Autònoma de Barcelona, 08193 Bellaterra, Barcelona, Catalonia, Spain

E-mail address: jllibre@mat.uab.cat

2 Département de Mathématiques, Centre Universitaire de Bordj Bou Arréridj, Bordj Bou Arréridj 34265, El anasser, Algeria

E-mail address: salhi3tayeb@yahoo.fr 